OFFICIAL ORGAN OF THE RADIATION RESEARCH SOCIETY

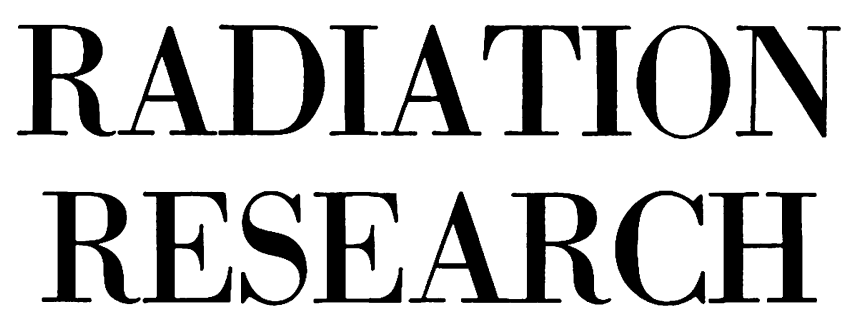

MANAGING EDITOR: TITUS C. EVANS

Volume 47, 1971

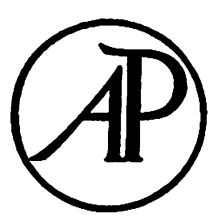

Academic Press - New York and London 
Copyright @ 1971 , by Academic Press, Inc.

\section{ALL RIGHTS RESERVED}

\footnotetext{
No part of this volume may be reproduced in any form by photostat, microfilm, by retrieval system, or any other means, without written permission from the publishers.
}

Made in the United States of America 


\section{RADIATION RESEARCH}

OFFICIAL ORGAN OF THE RADIATION RESEARCH SOCIETY

BOARD OF EDITORS: Managing Editor:

G. E. And.ss, Mt. Vernon Hospital, England

MI. J. Berger, National Bureau of Standards

J. (i. Carlsor, University of Tennessee

R. A. Conarid, Brookhaven National I aboratory

S. B. Curtss, Lniversity of California

W. Gor1)r, Duke University

D. Graнs, Argonne National Laboratory

M. I. Griem, University of Chicago

R. H. Hayses, York University, Caniada
Titus C. Evans, College of Medicine, University of Iowa, Iowa City, Iowa :)2:20

J. JAGGER, University of Texas

R. H. Johrses, llorida State University

R. F. Kalluan, Stanford University

S. Kondo, Osaka University, Japan

T. J. Phillips, University of California

P. Riesz, National Institutes of Health

W. C. Snipes, Pennsylvania State University

H. D. Surt, University of Texas

J. K. Thonas, University of Notre Dame

L. J. Tolmach, Washington University G. M. Woonwell, Brookhaven National Iaboratory

OFFICERS OF THE SOCIETY: President, N. F. BARR, Atomic Energy Commission, Washington, D.C.

Vice President (and President Elect), A. D. Conger, Temple University, Philadelphia, Pennsylvania.

Secretary-Treasurer, Falconer Simth, Biology Department, American University, Washington, D. C. 20016

Mamaging Editor, Titus C. Evans, University of Iowa, Iowa City, Iowa

Executive Secrefary: Richard J. Burk, JR., 4211 39th Street, N. W., Washington, D. C. 20016

Assistant to Managing Editor: Dorothy D. Schotrelius, University of Iowa, Iowa City, Iowa

\section{ANNUAL MEETINGS:}

197:2: May 14-18, Portland, Oregon

1973: April 29-May 3, St. Louis, Missouri

1974: July 13-20, V International Congress Radiation Research, Seattle, Washington

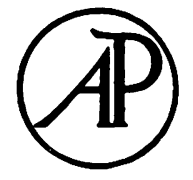


Councilors Radiation Research Society 1971-1972

\section{Physics}

R. J. Shalek, University of Texas

M. Ter-Pogossian, Washington University

\section{BIOLOGY}

R. F. Kallman, Stanford University

S. Wolff, University of California

\section{Medicine}

V. P. Bond, Brookhaven National Laboratory

L. K. Bustad, University of California

\section{Chemistry}

Jack Schubert, University of Pittsburgh

J. Weiss, Brookhaven National Laboratory

\section{At-Large}

E. L. Alpen, Battelle Memorial Institute

G. F. Whitmore, Ontario Cancer Institute 


\title{
CONTENTS OF VOLUME 47
}

\author{
Number 1, July 1971
}

E. K. Hussmaxx and W. L. McLaughuin. Dose-Distribution Measurement of High-Intensity Pulsed Radiation by Means of Holographic Interferometry

Albricht M. Klillerer axi Harald H. Rossi. RBE and the Primary Mechanism of Radiation Action

A. Ressill Joxis. The Radiolysis of Aliphatic Carboxylic Acids: The Decarboxylation of Normal Acids at $38^{\circ}$

W. E. Fatconer, R. Salovey, W. A. Sunder, and L. G. Walker. Effects of Dose, Dose Rate, and Trace Impurities on $n$-Hexadecane Radiolysis

E. Lukášová . .ND E. PALEČkK. Changes in DNA Secondary Structure after X-Irradiation

A. Chaccucei, (i. Colombetti, A. M. Columbe, P. R. Crippa, F. Llanci, and (i. MonT.IGNoli. Influence of the Molecular Organization on Radical Yield of Organic Compounds

PaCL Miny. Dose Dependence of the Transfer of Radiation-Induced Free Radicals from DNA to Crsteamine

Kurt (i. Hof:ir . .ND Warter L. Hucihes. Radiotoxicity of Intranuclear Tritum, ${ }^{125}$ Iodine and ${ }^{131}$ lodine

Chiu and A. M. Rauth. A Comparison of the Sensitivity to Ultraviolet Light of Mouse

L Cells and Mouse Bone Marrow Cells Assayed in vitro

J. Thomas P.trne and EdWard I. Shaw. A Radiation Effect from Low Doses of Tritiated Thymidine or X-Rays

Joseph li. Trayor and Harole W. Casey. Five-Year Follow-up of Primates lixposed to $55 \mathrm{Mev}$ Protons

Rosidd F. Hagkmax, Curtis P. Sigdestad, and S. Leshi:R. Intestinal Crypt Survival 143 and Total and Per Crypt Levels of Proliferative Cellularity Following Irradiation: Fractionated X-ray Exposures

Ronalo F. Hagmann and S. Lesher. Intestinal Crypt Survival and Total and Per Crypt Levels of Proliferative Cellularity Following Irradiation: Age Response and Animal Lethality

Sor M. Michanison and Brasind F. Schrervik, Jk. Cardiopulmonary liffects of UpperBody X-Irradiation in the Dog

Roblits Regh, Mardis Wohlfromm, Andrl: Varma, Nancy Splenclir, and W. StanronD. A Reexamination of the Mouse Embryonic Radiation Cataract Studies

P. V. Vittorio, J. F. Whitfilid, . INi) R. H. Rixon. The Radioprotective and Therapeutic Effects of Imidazole and Erythropoietin on the Frythropoiesis and Survival of Irradiated Mice

W. H. Knospr, J. Blom, H. B. Ciolostein, and W. H. Crosis. Delayed Bone Marrow Aplasia in Rats Protected Against Lethal Irradiation by Allogeneic Marrow Transplantation

Marg.url: Wirnkr. Effects of X-Irradiation on Steroid Synthesis in the Human Placenta 213

K. R. Trafines, H. Ernst, K. I. Altman, and L. H. Hempelmann. The Effect of X-Irradiation on Collagen Metabolism in Polyvinyl-Sponge Granulomas 
Abstracts of Papers for the Nineteenth Ammual Meeting of the Radiation Research Society, Boston, Massachuset ts, May 9-13, 1971

\section{Numbler 2, August 1971}

Albrecht M. Krilerler. Considerations on the Random Traversal of Convex Bodies and Solutions for General Cylinders

Albrecht M. KrLlerkr. An Assessment of Wall Effects in Microdosimetric Measurements

Marie M. Grexan and Edmund S. Copleand. Structure-Function Studies of the Aminothiol Radioprotectants. Effect of Carbon Chain Length in Mercaptoethylamine Homologs-Mammalian Radioprotection

John Chrysochoos and David S. Shinabi. X-Radiolysis of Aqueous Erythrosin 392

R. Katz, B. Ackerson, M. Homayoonfar, and S. C. Sharma. Inactivation of Cells by Heavy Iron Bombardment

James Shafffer and Timothy Merz. A Comparison of Unscheduled DNA Synthesis, $D_{0}$, Cell Recovery, and Chromosome Number in $x$-Irradiated Mammalian Cell Lines

W. U. Shipley, M. M. Flkind, and W. B. Prather. Potentiation of X-Ray Killing by 5-Bromodeoxyuridine in Chinese Hamster Cells: A Reduction in Capacity for Sublethal Damage Accumulation

Howard B. Newcombe and John F. McGregor. Persistence of Heritable Changes in an Irradiated Rat Population After Cessation of the Exposures

F. F. Hahn, E. Goldstein, and D. L. Dengworth. Effect of Whole-Body X-Irradiation on Pulmonary Bactericidal Function

Thomas C. Whlbourne ind G. B. Spurr. Mechanism of Renal Acid Excretion Following $\mathrm{X}$-Irradiation of the Dog

Shindds Warrien and Olive Gates. The Induction of Leukemia and Life Shortening in Mice by Continuous Low-Level External Gamma Radiation

Y. N. Kabakov. Factors Influencing Clasmatosis in Vivo: Observation of Periodicity of

Clasmatosis in Lymphocytes and Hematopoietic Recovery After Irradiation
uo-PAO YANG AND OtTo W. Neuhaus. In Vilro Uptake of Model Amino Acids by Rat

Liver Following Whole-Body $\gamma$-Irradiation 500

C. T. GAFFEy. Relation Between RBE and LET to Inhibit Neural Impulse Conduction 511

JонN M. Yuнas. Biological Factors Affecting the Radioprotective Efficiency of S-2-13Aminopropylamino]Ethylphosphorothioic Acid (WR-2721): LD $50(7)$ Doses

Charles O. McKinney and Frederick B. Turner. Genetic Variation in Irradiated and Nonirradiated Populations of the Lizard, Ula slansburiana

John B. Storkir. Chemical Protection of the Mouse against Radiation-Induced Iife short ening

H. Yoshikura. Radiation Effects on Macromolecular Synthesis in Contact InhibitionSensitive Cells Synchronized by Medium Change

H. I. Popescu and D. T. Stafanescu. Cytogenetic Investigation of Industrial Workers Occupationally Exposed to Gamma Rays

Book ReviEw

ANNOUNCEMENT

\section{Number 3, September 1971}

Antonije Dulčıć and Janko N. Hsrak. Crystal Structural Dependence of RadiationInduced Radicals in Thymine: An ESR Study

T. W. Armstrong AND B. L. Bishop. Calculation of the Absorbed Dose and Dose Equivalent Induced by Medium-Energy Neutrons and Protons and Comparison with Experiment 
Staniey Kronenberg, Robert Lux, and Kristian Nilson. Relative Biological Effectiveness of X-Rays Delivered at Very High Dose Rates to Radish Seeds (Raphanus salivus)

P. C. Shragge, H. B. Michands, and J. W. Hunt. Factors Affecting the Rate of Hydrated Electron Attack on Polynucleotides

P. Neta and Robert H. Schuler. Rate Constants for Reaction of Hydrogen Atoms with Compounds of Biochemical Interest

M. H. Wy koff. Distribution of ${ }^{134} \mathrm{Cs}$ in the Conceptus of the Pregnant Rat

M. R. Raju, M. Gnanapurani, B. Stackler, B. I. Martins, U. Madhvanath, J. Howard, J. T. Lyman, AND R. K. Mortimer. Induction of Heteroallelic Reversions and Lethality in Saccharomyces cerevisiae Fxposed to Radiations of Various LET ${ }^{60} \mathrm{Co} \gamma$ Rays, Heavy Ions, and $\pi^{-}$Mesons) in Air and Nitrogen Atmospheres

Howard H. Vogel, JR. and Robert Zaldívar. Cocarcinogenesis: The Interaction of Chemical and Physical Agents

Bala LakChaURA and J. B. Clark. Recovery from X-Ray and Nitrogen Mustard Inactivation of Escherichia coli by 2,4-Dinitrophenol Treatment

W. C. Dewey, L. E. Stone, H. H. Miller, and R. E. Giblak. Radiosensitization with 5-Bromodeoxyuridine of Chinese Hamster Cells X-Irradiated During Different Phases of the Cell Cycle

Gary S. Shaber. Alteration of 'Taste Thresholds in the Rat Following Low Dose X-Irradiation

'Adolfo Portela, Marcelo Garfunkel, Jorge Vaccirt, Ana Maria Delbue, Peter A. Stewart, and Juan Carlos Periz. Radiation Effects on Water Permeability and Distribution in Frog Muscle Cells

IJ. L. Cooley and F. L. Miller, JR. Effects of Chronic Irradiation on Laboratory Populations of the Aquatic Snail Physa helerostropha

Lawrence: W. Davis and Thomas A. Strike. Effectiveness of Tranquilizing Drugs in Rats Exposed to Mixed Ciamma-Neutron Radiations

D. K. Myers. The Initial Effect of X-Radiation on Thymidine Incorporation into DNA in Thymus Cells

S. Phyllis Stearner and Emily J. B. Christian. Mechanisms of Acute Injury in the $\gamma$-Irradiated Chicken. In Vivo Studies of Dose Protraction on the Microvasculature

Book ReVIEW

ERRATUM 


\section{CONTENTS}

E. I. Hussmann and W. I. McLaughlin. Dose-Distribution Measurement of High-Intensity Pulsed Radiation by Means of Holographic Interferometry

Albrecht M. Kellerer and Harald H. Rossi. RBE and the Primary Mechanism of Radiation Action.

A. Russell Jones. The Radiolysis of Aliphatic Carboxylic Acids: The Decarboxylation of Normal Acids at $38^{\circ}$

W. E. Falconer, R. Salovey, W. A. Sunder, and I. G. Walker. Effects of Dose, Dose Rate, and Trace Impurities on n-Hexadecane Radiolysis

E. Lukášová and E. Paleček. Changes in DNA Secondary Structure after X-Irradiation

A. Checcucci, G. Colombetti, A. M. Columbu, P. R. Crippa, F. Lenci, and G. Montagnoli. Influence of the Molecular Organization on Radical Yield of Organic Compounds

Paul Milvy. Dose Dependence of the Transfer of Radiation-Induced Free Radicals from DNA to Cysteamine

Iurt G. Hofer and Walter L. Hughes. Radiotoxicity of Intranuclear Tritium, ${ }^{125}$ Iodine and ${ }^{131}$ Iodine

S. Chiu and A. M. Rauth. A Comparison of the Sensitivity to Ultraviolet Light of Mouse L Cells and Mouse Bone Marrow Cells Assayed in Vitro

J. Thomas Payne and Edward I. Shaw. A Radiation Effect from Low Doses of Tritiated Thimidine or $X$-Rays

Joseph E. Traynor and Harold W. Casey. Five-Year Follou-up of Primates Exposed to $55 \mathrm{Mev}$ Protons

Ronald F. Hagemann, Curtis P. Sigdestad, and S. Jesher. Intestinal Crypt Survival and Total and Per Crypt Levels of Proliferative Cellularity Following Irradiation: Fractionated X-ray Exposures

Ronald F. Hagemann and S. Lesher. Intestinal Crypt Survival and Total and Per Crypt Levels of Proliferative Cellularity Following Irradiation: Age Response and Animal Lethality

Sol M. Michaelson and Bernard F. Schreiner, Jr. Cardiopulmonary Effects of L Lper-Body X-Irradiation in the Dog

Roberts Rugh, Marlis Wohlfromm, Andre Varma, Nancy Spencer, and W. Stanford. A Reexamination of the Mouse Embryonic Radiation Cataract Studies

P. V. Vittorio, J. F. Whitfield, and R. H. Rixon. The Radioprotective and Therapeutic Effects of Imidazole and Erythropoietin on the Erythropoiesis and Survival of Irradiated Mice

H. Knospe, J. Blom, H. B. Goldstein, and W. H. Crosby. Delayed Bone Marrow Aplasia in Rats Protected Against Lethal Irradiation. by Allogeneic Marrow Transplantation

Margaret Wiener. Effects of X-Irradiation on. Steroid Synthesis in the Human Placenta

K. R. Traelnes, H. Ernst, K. I. Altman, and L. H. Hempelmann. The Effect of X-Irradiation on Collagen Metabolism in PolyvinylSponge Granulomas

Abstracts of Papers for the Nineteenth Annual Meeting of the Radi-
ation Research Society, Boston, Massachusetts, May 9-13, 1971

Abstracts of Papers for the Nineteenth Annual Meeting of the Radi-
ation Research Society, Boston, Massachusetts, May 9-13, 1971

Announcement

Published monthly at Mt. Royal \& Guilford Aves., Baltimore, Md. 21202, by Academic Press, Inc. 111 Fifth Avenue, New York, N.Y. 10003.

(C) 1971 by Academic Press, Inc.

All correspondence and subscription orders should be addressed to the office of the Publishers at 111 Fifth Ave., New York, N.Y. 10003.

Send notices of change of address to the office of the Publishers at least 4 weeks in advance. Please include both old and new addresses.

In 1971, Volumes 45-48 will be published. Price of each volume: $\$ 20.00$. 


\title{
An Assessment of Wall Effects in Microdosimetric Measurements ${ }^{1}$
}

\author{
ALBRECHT M. KELLERER \\ Radiological Research Laboratory, Columbia University, 630 West 168th Street, \\ New York, New York 10032
}

\begin{abstract}
ILellerer, Albrech', M. An Assessment of Wall Effects in Microdosimetric Measurements. Radiat. Res. 47, 377-386 (1971).

Proportional counters with solid walls exhibit distortions of pulse height distributions due to the so-called wall effects. With heavy charged particles these wall effects mainly affect the indirect events of energy deposition, i.e., those events where the heavy charged particle does not actually enter the cavity but merely injects some delta rays into it. The percentage of absorbed dose due to these distorted pulses is calculated for charged particles of different energy and for neutron fields. For neutrons below $10 \mathrm{M} \mathrm{IeV}$ the wall effects are generally small, and conventional proportional counters are applicable.
\end{abstract}

\section{INTRODUCTION}

Microdosimetric measurements performed with tissue equivalent proportional counters exhibit inaccuracies due to the so-called wall effects. These wall effects have been discussed by Failla (1) and by Rossi (2); one can state in general that they distort the observed spectra of energy deposition and cause a certain shift of the spectra towards larger values.

Proportional counters applied in microdosimetry are commonly filled with gas of the same atomic composition as the surrounding walls. In a first approximation one can therefore assume that the collision cross sections for charged particles are the same in the gas and in the wall material. Under this assumption the particle tracks in the gas alone are equal to the tracks in the condensed material except for a scaling factor inversely proportional to the ratio of the densities.

Under suitable conditions one obtains a uniform radiation field throughout the counter. The fluence spectrum is then constant, and track segments of various shapes occur with the same relative frequency within the cavity and the simulated

${ }^{1}$ Based on work performed under Contract AT-(30-1)-2740 for the U.S. Atomic Energy Commission. 
region of the condensed material. The only effect of the walls is that some track segments in the cavity occur simultaneously while in the simulated domain they are statistically independent. This leads to the fact that a certain number of absorption events are eliminated, and some spurious larger events are induced.

With the widening application of microdosimetric techniques to the determination of radiation quality so-called wall-less counters (2) have found special interest. Rossi and co-workers have developed various types of wall-less counters; and yspectra obtained with such instruments by Gross ( 3 ), as well as those obtained by Glass and Braby (4), exhibit characteristic differences to results obtained with conventional equipment. It is, however, not easy to judge whether for any particular radiation and any particular size of the simulated tissue domain application of wallless counters is indicated, and, if so, what errors are involved if conventional counters are used. For this reason it is desirable to find a criterion which permits an approximate assessment of wall effects. The present paper deals with such a criterion for neutron fields and heavy charged particles.

\section{WALL EFFECTS WITH HEAVY CHARGED PARTICLES}

In most microdosimetric measurements the simulated tissue regions are of a size not exceeding several micrometers. In such a region simultaneous occurrence of two charged particles individually set in motion by one and the same uncharged particle is unlikely; the tracks of the charged particles are in general too widely separated from each other. This is particularly true for neutron recoils. The collision cross sections for neutrons are nearly constant over a wide range of energies, and the typical mean free path for the neutron is of the order of magnitude of centimeters in tissue.

Wall effects are therefore due to the simultaneous occurrence of track segments belonging to or originating from the same charged particle track. If all charged particle tracks were straight lines, and if the radial extension of delta rays around the track core could be neglected there would be no wall effects in proportional counters.

For heavy charged particles the first condition is very nearly fulfilled, if one neglects nonelastic scattering of neutrons which is insignificant below $10 \mathrm{MeV}$ and spallation which need not be considered below neutron energies of $100 \mathrm{MeV}$. The radial extension of the delta ray halo around the track of the heavy recoil particles is, however, in many cases comparable to the dimensions of the simulated tissue region, and this is the main factor responsible for wall effects.

The following discussion will, as far as neutron fields are concerned, be restricted to recoil protons because the extension of delta ray tracks is much less for the heavier recoils due to the smaller velocities of the heavier recoils. Wall effects due to the V-shaped particle tracks generated in nonelastic scattering and due to the stars of particle tracks generated in spallation are significant only at high energies. Some remarks on these contributions will be made at the end of the paper.

When a microscopic tissue region is exposed to a field of heavy charged particles, 
one can distinguish two kinds of events of energy deposition. The heavy charged particle itself can traverse or partly traverse the region; the energy deposition event will then be called a direct event. On the other hand, the heavy charged particle can pass outside the region but inject one or several delta rays into it; one may then speak of an indirect event. Figure 1 gives a schematic representation of the two types of events in the practically important case of a spherical region.

If the tissue region is simulated by a cavity the direct events are not significantly changed as indicated in the scheme of Fig. 2a. There is a somewhat increased probability that delta rays leaving the region will reenter this region, and some delta rays created outside the cavity can enter it while in the actual situation they would miss the region. But the energy involved in these wall effects represents a small fraction of the total energy involved in the direct event. One may therefore assume that the representation of direct events in a conventional counter is nearly correct.

Indirect events, however, are not correctly represented in a cavity. If a charged particle passes outside the reference region within a distance small enough that its delta rays can reach this cavity, then a much larger piece of its track is close to the region of interest than in the actual case of a microscopic tissue region. This fact which is schematically represented in Fig $2 \mathrm{~b}$ reflects the fact that the radius of curvature as seen from outside the cavity is much larger than the radius of curvature of the simulated region.

The resulting effect is that for the cavity the indirect events are strongly distorted; the average number of delta rays injected in an indirect event is far greater

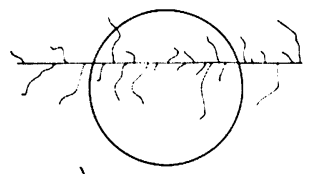

a)

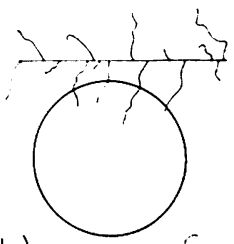

b)

FIG. 1. Schematic representation of (a) direct and (b) indirect events in a homogeneous medium.

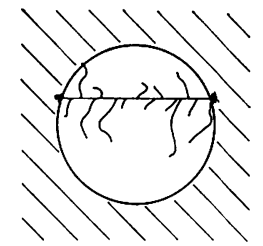

a)

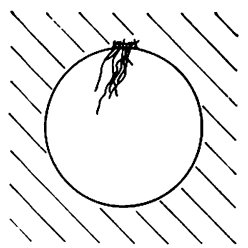

b)

FIG. 2. Schematic representation of (a) direct and (b) indirect events in a cavity. 
than for the simulated region. On the other hand, the relative contribution of delta ray influx to the absorbed dose must be equal for a cavity and the corresponding tissue region. This follows from the fact that the fluence and fluence distribution is constant under the conditions of Fano's theorem (5). Thus the walls have the effect that most of the indirect events are suppressed. Their contribution is compensated by an increased delta-ray influx in the rest of the events. Experimental studies with wall-less counters by Gross et al. (3) have indeed demonstrated a significant number of small indirect events unobserved with conventional counters.

One concludes that conventional counters can be applied whenever the indirect events represent a negligible fraction of the absorbed dose, and it remains to be seen how this fraction depends on the nature of the radiation field and on the size of the simulated region.

\section{THE FRACTION OF ENERGY INVOLVED IN INDIRECT EVENTS}

As a first step in the calculation one may derive the probability that energy deposition at a given distance $x$ from the track core belongs to an indirect and not a direct event when one deals with a spherical region $R$ of diameter $d$.

Figure 3 can assist in visualizing the geometry for a solution of this problem. The point $P$ represents the location of energy deposition at distance $x$ from the track core. The track core is depicted by the straight line $L$. The dotted circle of diameter $d$ represents the spherical domain which comprises all those positions of the center of the region of interest $R$ for which $P$ lies inside $R$. The two broken lines symbolize the outline of a cylinder of diameter $d$ around $L$. This cylinder contains all positions of the center of $R$ for which $L$ passes through $R$. The probability that the energy deposition represented by the point $P$ belongs to an indirect event is determined by the fraction of the volume of the spherical domain in Fig. 3 which lies outside the cylinder. The determination of this fraction as a function of the ratio $b=x / d$ is an elementary geometrical problem. The volume of overlap can be derived by integrating it in pieces of cylindrical shells whose axis goes through the point $P$ and is parallel to the line $L$. This leads to the following integral for the fraction $P(b)$ of the volume which does not overlap:

$$
P(b) \quad 1-\frac{3}{\pi} \int_{0}^{1} r \sqrt{1-r^{2}} f(r) d r \quad 0<b<1
$$

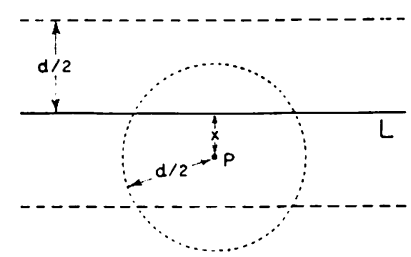

Frg. 3. Determination of the probability that energy deposition at a distance $x$ from the track core is involved in an indirect event. 
with

$$
f(r)= \begin{cases}\pi & \text { for } r<1-2 b \\ 0 & \text { for } r<2 b-1 \\ \cos ^{-1} \frac{r^{2}+4 b^{2}-1}{4 b r} & \text { otherwise. }\end{cases}
$$

The integral has been evaluated numerically and the resulting probability $P(b)$ for indirect events is plotted in Fig. 4 . With this function and with a knowledge of the radial distribution of energy around the track core one can calculate the fraction $\rho(d)$ of energy - or absorbed dose-which is due to indirect events in a spherical region of diameter $d$.

Consider a heavy charged particle with kinetic energy $E$ per nucleon. The distribution of energy deposition in distance from the track core is $\mathbf{d}(x ; E)$. The kinetic energy $E$ of the particle is given as a parameter because the distribution refers to a track segment traversed at this particular energy. One then has the solution:

$$
\rho(d)=\int_{0}^{\infty} P(x / d) \mathbf{d}(x ; E) d x
$$

In the case of irradiation with neutrons of a given energy $E_{0}$ one must integrate over all energies between 0 and $E_{0}$. One may neglect all heavy recoils and merely consider protons since they have the highest velocity and therefore the most significant radial extension of delta rays. To a good approximation the initial energy of the recoil protons is distributed equally between 0 and $E_{0}$. The fraction of energy deposition due to particles of kinetic energy between $E$ and $E+d E$ is therefore equal to $2\left(1-E / E_{0}\right) d E$, and one obtains

$$
\rho(d)=\int_{0}^{\infty} P(x / d) \mathbf{d}(x) d x
$$

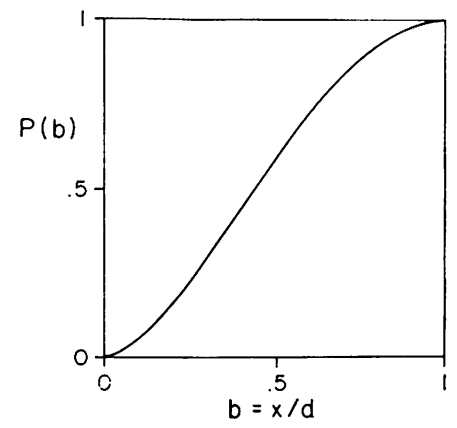

FIG. 4. Probability that energy deposition at distance $x$ from the track core belongs to an indirect event in a spherical region of diameter $d$. 
with

$$
\mathbf{d}(x)=2 \int_{0}^{E_{0}}\left(1-E / E_{0}\right) \mathbf{d}(x ; E) d E
$$

The numerical evaluation of these expressions is straightforward, but knowledge of the radial energy distribution $\mathbf{d}(x ; E)$ as a function of the kinetic energy $E$ is limited. A theoretical evaluation of this function on the basis of available collision cross sections is difficult, not so much because the cross sections are poorly known, but because the calculations have to include such complicating factors as energy loss straggling and directional straggling of the delta rays. Monte Carlo methods seem to be most promising in this respect but have not been performed at present. Several authors (6-8) have performed calculations under simplified assumptions. Wingate and Baum ${ }^{2}$ have obtained experimental results over a limited range of proton energies. The results of the different authors are in approximate agreement with each other and with the experimental results. Figure $5 \mathrm{a}$ is derived from the experimental data (7). The curves represent sum distributions belonging to the differential distributions discussed above. $\mathbf{D}(x ; E)$ is the fraction of the energy laid down at a distance larger than $x$ from the track core of a heavy particle of energy $E$ per nucleon.
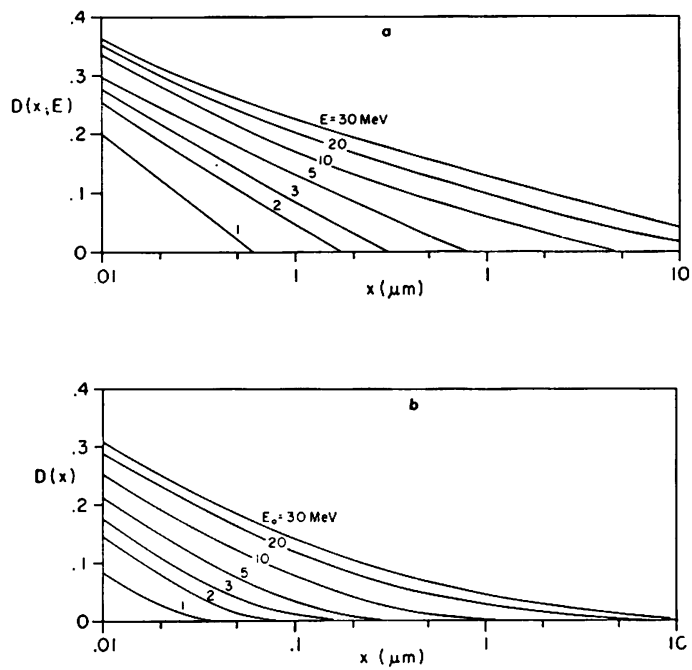

FIG. 5a. Fraction of absorbed dose delivered at a distance greater than $x$ from the track core of a particle at energy $E$ per nucleon. The curves are derived from data given in (7).

FIg. 5b. Fraction of absorbed dose delivered at a distance greater than $x$ from the track core for recoil protons generated by neutrons of energy $E_{0}$.

${ }^{2}$ C. L. Wingate and J. W. Baum, Micro-radial distributions of dose and LET for alpha and proton beams. Doc. No. BNL 14767, Brookhaven (1970). 
In terms of the sum distributions which are more convenient in the actual numerical evaluation Eqs. (2)-(4) take the form:

$$
\rho(d)=\int_{0}^{\infty} P(x / d) d \mathbf{D}(x ; E)
$$

and

$$
\rho(d)=\int_{0}^{\infty} P(x / d) d \mathbf{D}(x)
$$

with

$$
\mathbf{D}(x)=2 \int_{0}^{E_{0}}\left(1-E / E_{0}\right) \mathbf{D}(x ; E) d E .
$$

The solutions of Eq. (7), that is, the radial profiles of the track integrated over the total spectrum of proton recoils from monochromatic neutrons, are represented in Fig. 5b.

\section{RESULTS}

Figure 6a represents the fraction $\rho(d)$ of absorbed dose contributed by indirect events in a spherical tissue region of diameter $d$. The parameter is the linetic energy $E$ per nucleon of the charged particle. Because these curves refer to Eq. (5), i.e., to a fixed energy, they are applicable under the conditions of the so-called track segment experiments.
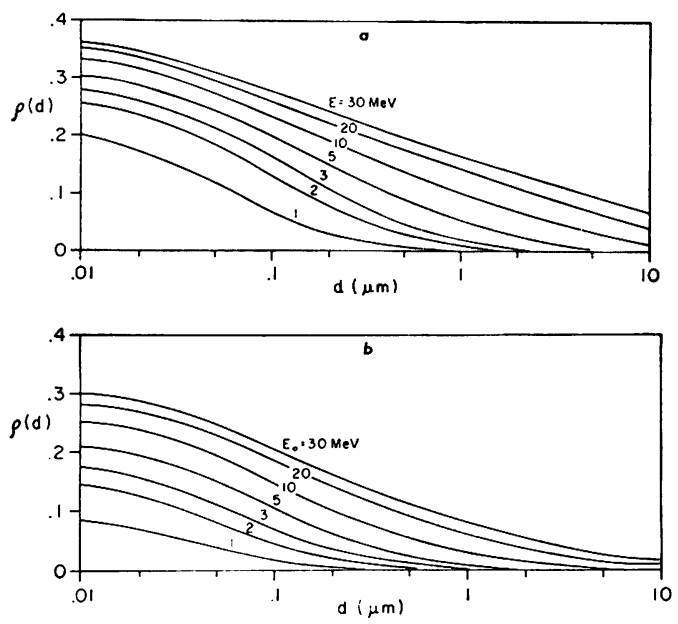

FIG. 6a. Fraction $\rho(d)$ of absorbed dose due to indirect events in spherical tissue regions of diameter $d$. The curves refer to track segments of heavy particles with kinetic energy $E$ per nucleon.

FIG. 6b. Fraction $\rho(d)$ of absorbed dose due to indirect events in spherical tissue regions of diameter $d$. The curves refer to the proton recoils of neutrons of energy $E_{0}$. 
Microdosimetric measurements with spherical wall-less counters as described by Gross (3) could be used to derive the quantity $\rho(d)$ experimentally for different values of $E$. Comparison with the results given in Fig. 6a stould then provide a check of the validity of the energy profiles represented in Fig. 5a.

Figure $6 \mathrm{~b}$ depicts the results for fields of monoenergetic neutrons, according to Eq. (3). The fraction $\rho(d)$ of absorbed dose due to indirect events is given as a function of the diameter $d$ of the spherical tissue region. The parameter is the energy $E_{0}$ of the neutrons.

The calculations are based only on the proton recoils. The heavier recoils have very short delta rays and contribute almost nothing to the indirect events. They are therefore not involved in wall effects except in the case where several heavy charged particles start from the same point as in nonelastic neutron scattering or in spallation processes. Spaliation plays no role in the energy range considered here and nonelastic scattering is significant only for energies above $10 \mathrm{MeV}$. An analysis of the wall effects due to the $\mathrm{V}$-shaped tracks in nonelastic events has been outlined by Biavati. ${ }^{3}$. Ionte Carlo calculations performed by Oldenburg and Borz (9) have verified that the effect is negligible for a neutron energy of $6.5 \mathrm{MeV}$. Though the fractional energy involved in these wall effects is small and the frequency mean and energy mean of the $y$-spectra are not significantly affected, one should note that for neutron energies exceeding $10 \mathrm{MeV}$ the tail end of the $y$ distributions can be distorted. This can be important in considerations involving the exact shape of the $y$ distributions in the region of largest values of $y$.

While wall effects due to the heavier recoils have some influence above $10 \mathrm{MeV}$, a correction of the curves for 20 and $30 \mathrm{MeV}$ appears unwarranted in view of the uncertainty of the input data represented in Fig. 5a. For the same reason it is unnecessary to refer the curves in Fig. 6 to the total dose delivered by heavy recoils instead of referring it to the proton contribution alone which is roughly $10-20 \%$ less.

The practical applicability of the results is not strongly affected by the inaccuracy of the input data because $\rho(d)$ varies slowly with $d$. If one wants to obtain a highly. conservative estimate of the relative contribution of indirect events one can assume that the energy profiles around the particle tracks have twice the radial extension represented in Fig. 5. One must then read Fig. 6 at half the value of $d$.

One concludes from the data of Fig. $6 \mathrm{~b}$ that conventional tissue equivalent counters are quite suitable for most microdosimetric measurements in neutron fields. These counters do not properly represent the indirect events in which a proton does not actually pass through the sensitive volume but merely injects some of its delta rays. At an equivalent diameter of $1 \mu \mathrm{m}$ the contribution of indirect events to absorbed dose varries between 3 to $8 \%$ for neutron energies between 10 and $30 \mathrm{MeV}$. For lower neutron energies the contributions are still smaller.

${ }^{3}$ B. J. Biavati, Frequency of multiple tracks in equivalent cavities of varying density. Annual Report on Research Project, Doc. No. NYO-2740-3, U.S. Atomic Energy Comm. (1966). 
The application of conventional counters is particularly well justified in radiation protection applications. The wall effects consist of a substitution of a number of small indirect events by a few larger events. The small errors incurred in the determination of quality factors are therefore conservative.

One should note that neutron fields are never completely free of a $\gamma$-ray component. Wall effects due to the $\gamma$-ray component are not covered in the preceding discussion.

One must also notice that the fraction of energy involved in distorted pulses is just one of several possible criteria for the extent of wall effects. The fractional number of pulses which are not properly represented in conventional counters can be considerably larger because the indirect pulses belong to the low end of the y-spectrum. ${ }^{4}$ Accordingly the frequency mean $\bar{y}_{F}$, of the $y$-spectra can be shifted appreciably even in those cases where the energy mean $\bar{y}_{D}$ is hardly affected. $\bar{y}_{D}$ is the analogue of the dose mean LET, $\bar{L}_{D}$, and therefore in many cases the relevant index of radiation quality. There are, however, radiobiological applications of microdosimetric data which involve event frequency rather than the distribution of energy involved in the energy deposition events. The most notable examples are effects of very heavy nuclei. For these particles the track core exhibits such a high LET that most of its energy is wasted. The indirect events can then be of great importance, and the determination of $y$-spectra with wall-less proportional counters is essential.

The preceding discussion has been concerned with the inaccuracies involved in the application of conventional proportional counters in microdosimetry. One may observe that the result can as well be used to judge the validity of the theoretical derivation of microdosimetric spectra according to the method developed by Caswell (11). This method is based on the fact that energy straggling and the radial extension of the tracks of neutron recoils can be neglected if the region of interest is large enough and if the energy of the recoils is moderate.

Figure $6 \mathrm{~b}$ indicates the error involved in the neglection of the finite radial extension of the tracks. One concludes that, as far as this factor is concerned, the calculations according to Caswell's method are as valid as are results obtained with conventional counters. For neutron energies up to a few $\mathrm{MeV}$ the method may be applied even to regions considerably smaller than $1 \mu \mathrm{m}$ in diameter.

Formulae for the influence of energy loss straggling on the microdosimetric distributions have been given earlier (12). One finds that the factor is significant in the same range of neutron energies and site diameters in which the radial extension of the tracks is critical.

\section{ACKNOWLEI)(IMENTS}

I am grat eful to I)r. H. H. Rossi and D)r. W. Gross for various suggestions. Dr. (ross has given valuable help in the numerical evaluations.

ReCEIVED: January 25, 1971

${ }^{4}$ For a definition of microdosimetric quantities see (10). 


\section{REFERENCES}

1. P. FaILla and G. FallLa, Measurement of the dose in small tissue volumes surrounding "point" sources of radioisotopes. Radiat. Res. 13, 61-91 (1960).

2. H. H. Rossi, Microscopic energy distribution in irradiated matter. In Radialion Dosimetry (F. H. Attix and W. C. Roesch, eds.), Vol. 1, pp. 43-92. Academic Press, New York (1968).

3. W. Gross, B. J. Biavati, and H. H. Rossi, Microdosimetry of directly ionizing particles with wall-less proportional counters. In Microdosimetry, Proceedings of 2nd Symposium on Microdosimetry (H. G. Ebert, ed.), pp. 249-267. Euratom, Brussels (1969).

4. W. A. Glass and L. A. Brais , A wall-less detector for measuring energy deposition spectra. Radial. Res. 39, 230-240 (1969).

5. U. FAno, Note on the Bragg-Gray cavity principle for measuring energy dissipation. Radial. Res. 1, 237-240 (1954).

6. R. KAtz, Particle tracks in emulsion. In Microdosimetry, Proceedings of 2nd Symposium on Microdosimetry (H. G. Ebert, ed.), pp. 631-652. Euratom, Brussels (1969).

7. J. W. BAUM, Comparison of distance- and energy-restricted linear energy transfer for heavy particles with 0.25 to $1000 \mathrm{MeV} / \mathrm{AMU}$. In Microdosimetry, Proceedings of 2nd Symposium on Microdosimetry (H. G. Ebert, ed.), pp.653-666. Euratom, Brussels (1969).

8. H. Paretzke and G. Burger, Spatial distribution of deposited energy along the path of heavy charged particles. In Microdosimetry Proceedings of 2nd Symposium on Microdosimetry (H. G. Ebert, ed.), pp. 615-630. Euratom, Brussels (1969).

9. U. Oldenburg and J. Booz, Wall effects of spherical proportional counters. In Microdosimelry, Proceedings of 2nd Symposium on Microdosimetry (H. G. Ebert, ed.), pp. 269-281. Euratom, Brussels (1969).

10. A. M. Kellerer and H. H. Rossi, Summary of quantities and functions employed in microdosimetry. In Microdosimetry, Proceedings of 2nd Symposium on Microdosimetry (H. G. Ebert, ed.), pp. 843-853. Euratom, Brussels (1969).

11. R. S. Caswell, Deposition of energy by neutrons in spherical cavities. Radial. Res. 27, 92-107 (1966).

12. A. M. Kellerer, Analysis of patterns of energy deposition. In Microdosimetry, Proceedings of 2nd Symposium on Microdosimetry (H. G. Ebert, ed.), pp. 107-135. Euratom, Brussels (1969). 